\title{
CHARGE TRANSFER COMPLEXES IN ITRACONAZOLE
}

\author{
KUCHARLAPATI. DEEPTHI
}

Research Scholar

HYDERABAD, Telangana

\begin{abstract}
Itraconazole (ITZ) is a distinguished antifungal representative that also has anticancer commotion. In this study, we recognize ITZ as a wide-ranging inhibitor of enteroviruses (e.g., poliovirus, coxsackievirus, enterovirus-71, rhinovirus). We exhibit that ITZ reduces viral RNA duplication by aiming oxysterol-binding protein (OSBP) and OSBP-related protein 4(ORP4). Time after time, OSW-1, a precise OSBP/ORP4 antagonist, too restrains enterovirus replication. Giveaway of OSBP inhibits virus replication, while over expression of OSBP or ORP4 offsets the antiviral belongings of ITZ and OSW-1. ITZ binds OSBP and inhibits its purpose, i.e., shuttling of cholesterol and phosphatidylinositol-4-phosphate between membranes, thus likely disturbing the virus-induced membrane alterations are also indispensable for viral replication organelle construction. ITZ also inhibits hepatitis $\mathrm{C}$ virus replication, which also relies on OSBP. Together, these data implicate OSBP/ORP4 as molecular targets of ITZ and point to an imperative utility of OSBP/ORP4-mediated lipid barter in virus replication that can be besieged by antiviral meds.
\end{abstract}

Keywords: - drug, human, infections, membranes, proteins, virus

\section{INTRODUCTION}

The family Picornaviridae encloses a lot of significant human being and animal pathogens. The genus Enterovirus embraces poliovirus (PV), coxsackievirus (CV), echovirus, numerous numbered enteroviruses (e.g., enterovirus-71 [EV71]), and human rhinovirus (HRV). Apart from for PV, no vaccines are accessible to put off contagions with enteroviruses and no antiviral meds are presented for healing.
Additional significant human picornaviruses comprise hepatitis A virus and human parechovirus $(\mathrm{HPeV})$. Eminent animal pathogens are foot-and-mouth ailment virus and encephalo-myocarditis virus (EMCV).

The genome of enteroviruses consists of a $7.5 \mathrm{~kb}$ single- stranded RNA molecule of affirmative polarity [(+) RNA]. It encodes a sole polyprotein that is 


\section{(INTERNATIONAL JOURNAL ONLINE OF SCIENCE)}

\section{ISSN: $2455-0108$}

proteolytically processed by the viral proteases into the structural proteins (VP1VP4) and the nonstructural proteins (2A-2C and $3 \mathrm{~A}-3 \mathrm{D})$. The viral genome is simulated by assemblies of viral and host proteins to be found on intracellular membranes termed replication organelles (ROs). The ROs are fashioned as an outcome of virusinduced remodeling of secretory pathway membranes, which presumably starts at the Golgi complex (Hsu et al., 2010), finally ensuing in a multifaceted network of tubulovesicular membranes (Belov et al., 2012; Limpens et al., 2011). Viral alteration of lipid homeostasis is thought to play a major function in RO configuration. Viral proteins $2 \mathrm{BC}$ and $3 \mathrm{~A}$ take part in a foremost part in the membrane rearrangements by recruiting necessary host aspect for enterovirus replication to ROs, such as phosphatidylinositolphosphate-4-kinase III beta (PI4KIIIb), a Golgi-localized lipid kinase that engenders phospha- tidylinositol-4 phosphate (PI4P) (Arita, 2014; Hsu et al., 2010). The purposeful significance of eminent PI4P levels at ROs remains to be recognized. The viral RNA-dependent RNA-poly-merase, 3Dpol, binds PI4P in vitro, but it is unidentified whether this is significant for its conscription and/or commencement in infected cells (Hsu et al., 2010). Instead, the PI4P lipids may contribute in RO formation by facilitating the conscription of PI4Pbinding host proteins with membranemodifying features.

Cholesterol is a decisive membrane element that decides membrane fluidity and normalizes the formation and occupation of
Volume II, Issue VI SEPTEMBER

membrane bound complexes of lipids and proteins. A number of (+) RNA viruses, such as hepatitis $\mathrm{C}$ virus (HCV), dengue, and West Nile virus, alter the cellular cholesterol scene to make intracellular host-cell membranes favorable for competent genome replication (Rothwell et al., 2009; Wang et al., 2014). Enterovirusinduced rearrangements of secretory pathway membranes into the tubulovesicular RO structures may also depend on revisions in cholesterol homeostasis. Recent data advocates that enteroviruses encourage clathrin-mediated endocytosis to transport cholesterol from the plasma membrane and extracellular medium to ROs (Ilnytska et al., 2013). Though, further intracellular cholesterol trafficking trails may also be undermined by enteroviruses to create their ROs.

In recent times, oxysterol-binding protein (OSBP) was exposed to play a key function in the transport of cholesterol and PI4P amid the endoplasmic reticulum (ER) and Golgi (Mesmin et al., 2013). OSBP links ER and trans-Golgi membranes at ostensible ER- Golgi membrane contact ites (MCSs) and shuttles sterol into the Golgi and PI4P back to the ER, where it is hydrolyzed by the PI4P-phosphatase Sac1. This cholesterol/PI4P exchange cycle constrains the release of sterol in the transGolgi and self-regulates the localization of OSBP on the Golgi. OSBP and the OSBPrelated proteins (ORPs) compose a family of connected proteins that, derived from gene structure and succession, can be subdivided into six subfamilies. OSBP and its contiguous relative, ORP4 (also called 


\section{(INTERNATIONAL JOURNAL ONLINE OF SCIENCE) \\ ISSN: $2455-0108$ \\ Volume II, Issue VI SEPTEMBER}

OSBP2), fit in to subfamily I. All ORPs have a lipid-binding domain that was primarily contemplated to be precise for sterols. However, current structural analysis recommends that ORPs have the capability to fasten PI4P and a second lipid that is either a sterol or a nonsterol ligand. Numerous ORPs, including OSBP, encompass an FFAT-motif that is known by ER-resident VAP receptors and an $\mathrm{N}$ terminal pleckstrin homology $(\mathrm{PH})$ domain for fastening PI4P, throughout which they are linked to a range of organelles. Though the functions of most ORPs are not very well understood, it has turn into understandable that ORPs carry out assorted functions in lipid sensing, lipid transport, and cell signaling (Raychaudhuri and Prinz, 2010; Weber-Boyvat et al.,2013).

We commence to recognize original inhibitors of enterovirus replica-tion by showing the NIH Clinical Collection (NCC), a documentation of US Food and Drug Administration (FDA) accepted meds that have a record of use in clinical trials for cure of a large range of maladies. Related assortments of FDA-approved meds have confirmed to be affluent sources of undiscovered bioactivity and healing prospective. We recognized itraconazole (ITZ) as a wide-ranging inhibitor of enterovirus replication. ITZ is a distinguished antifungal medicine that inhibits CYP51, a cytochrome P450 necessary for sterol biosynthesis (Lestner and Hope,2013). Besides, ITZ puts forth anticancer commotion by inhibiting angiogenesis - throughout disquieting mTOR signaling and vascular endothelial augmentation aspect receptor 2 (VEGFR2) trafficking and the Hedgehog (Hh) signaling pathway (Kim et al., 2010; Nacev et al., 2011; Xu et al., 2010). ITZ has been found to be efficient in patients with numerous malignancy types in manifold stage 2 clinical studies (Antonarakis et al., 2013; Kim et al., 2014; Rudin et al., 2013). We exhibit that acknowledged targets of ITZ cannot elucidate the antiviral action of ITZ. Instead, confirmation is presented that OSBP and ORP4 are narrative targets of ITZ and that straight binding of ITZ to OSBP, which localizes at ROs, disrupts its lipid-shuttling role, and accounts for the antiviral result of ITZ.

A screen of the NCC is performed to identify novel inhibitors of CVB3 replication. Similar to all enteroviruses, CVB3 kills its host cell and thereby causes a "cytopathic effect" (CPE). We screened the NCC by microscopically observing which compounds prevented the development of CPE in a multicycle replication assay and identified ITZ as one of the hits. To determine its spectrum of antiviral activity, we tested ITZ against a representa- tive panel of picornaviruses in a multicycle CPE-reduction assay. ITZ exhibited antiviral effect against all enteroviruses examined (belonging to several species) with $50 \%$ effective concentration (EC50) values between 0.3 $\mathrm{mM}$ and $1.6 \mathrm{mM}$. In addition, EMCV, a Cardiovirus genus member, was inhibited by ITZ. In contrast, equine rhinitis a virus (ERAV; Aphthovirus genus member) and HPeV-1 (Parechovirus genus member) were 


\section{(INTERNATIONAL JOURNAL ONLINE OF SCIENCE)}

\section{ISSN: $2455-0108$}

insensitive to ITZ. To exclude the possibility that the antiviral activity was due to toxic side effects, we determined the effect of ITZ on virus production during a single replication cycle. Similar to the multi- cycle CPE-reduction assay, ITZ was active against CVB3, EV71, HRV14, and EMCV, but not ERAV, in a single replication cycle without apparent toxicity. ITZ also inhibited Saffold virus (SAFV) replication, a human cardiovirus. Thus, ITZ exerts broad antiviral activity against en- teroviruses and cardioviruses.

\subsection{ITZ Inhibits Viral RNA Genome} Replication

Next, we decided the impact of ITZ on interpretation and replication of transfected CVB3 and EMCV RNAs, specifically a subgenomic replicon of CVB3, in which (part of) the capsidcoding locale is supplanted by a firefly luciferase quality or a genomic RNA of EMCV, in which a Renilla luciferase quality is embedded upstream of the coding area. As positive controls, we utilized guanidine-HCl and dipyridamole, understood and powerful inhibitors of CVB3 and EMCV replication, individually. Two hours after transfection of the RNAs, when no RNA replication has occurred yet (van Kuppeveld et al., 1995), luciferase levels were unaffected, indicating that ITZ does not hinder viral genome interpretation. In any case, at later time focuses, luciferase generation by both replicons was diminished, showing that ITZ influences RNA replication. Critically, ITZ did not influence viral polyprotein blend and preparing.
Volume II, Issue VI SEPTEMBER

2.2 Inhibition of Virus Replication Is Independent of Known Targets of ITZ

ITZ is broadly utilized as an antifungal medication that represses the contagious protein CYP51. ITZ has likewise been appeared to have some inhibitory movement toward the human CYP51 (hCYP51) and the related cytochrome P450 CYP3A4. Notwithstanding ITZ, other azole family antifungal meds, including posaconazole, ketoconazole, flucon-azole, and voriconazole (Figure S1), likewise hinder hCYP51 and CYP3A4 with somewhat lower or comparative intensity as ITZ (Warrilow et al., 2013; Zhang et al., 2012). We tried whether these meds apply antiviral movement utilizing recombinant infections RLuc-CVB3 and RLuc-EMCV, which convey the Renilla luciferase quality upstream of the coding area. At $10 \mathrm{mM}$, just posaconazole repressed replication of RLuc-CVB3 and RLuc-EMCV. The rest of the azoles did not show any antiviral action at fixations up to $100 \mathrm{mM}$. Comparable results were acquired in a multicycle CPEdiminishment test. These outcomes decided out the likelihood that hindrance of hCYP51 or CYP3A4 underlies the antiviral action of ITZ and its fundamentally related simple posaconazole.

As ITZ additionally represses the Hedgehog (Hh) flagging pathway, doubtlessly by meddling with the capacity of the G protein-coupled receptor-like protein Smoothened (Kim et al., 2010), we tried a few Smoothened rivals in the viral luciferase measures. The Smoothened rivals KAAD-cyclopamine, Sant-1, and Sant-2 (Chen et al., 2002; Taipale et al.,2000) had 


\section{ISSN: $2455-0108$}

no impact on the replication of RLuc-CVB3 or RLuc-EMCV, demonstrating that the antiviral movement of ITZ is not interceded by its restraint of the Hh pathway.

The antiangiogenic movement of ITZ has been ascribed in any event to some degree to its hindrance of the mTOR flagging pathway through interruption of the transporting of cholesterol between plasma layer and late endosomes/lysosomes, along these lines initiating the amassing of cholesterol in the endolysosomal framework (Xu et al., 2010). We observed that cholesterol, recolored with filipin, was redistributed by ITZ and posaconazole as well as by keto-conazole (which does not repress infection replication) in two human cell lines (HAP1 and HeLa R19 cells. Besides, the mTOR inhibitor rapamycin had no impact on picor-navirus replication (Beretta et al., 1996; Wong et al., 2008). Together, these outcomes propose that restraint of infection replication by ITZ or posaconazole is not because of interruption of endosomal cholesterol moving or the cholesterol-related mTOR hindrance. Notwithstanding the previously mentioned atomic and pathway focuses of ITZ, ITZ has been accounted for to bother $\mathrm{N}$ glycosylation(Nacev et al., 2011). Be that as it may, the $\mathrm{N}$-glycosylation inhibitor tunicamycin did not influence poliovirus (Doedens et al., 1997) or CVB3 replication. ITZ has additionally been appeared to threaten the estrogen receptor a (Time) (Cheng et al., 2012). In any case, Time agonist b-estradiol did not influence CVB3 or EMCV replication. At long last, ITZ has been accounted for to target $\mathrm{p}$-glycoprotein,
Volume II, Issue VI SEPTEMBER

UDP-glucuronosyltransferase, and ERb, none of which are liable to intervene the antiviral movement of ITZ, on the grounds that these are as strongly restrained by ketoconazole (Cheng et al., 2012; Walsky et al., 2012; Wang et al., 2002b), which did not influence infection replication.

Transformations in $3 \mathrm{~A}$ that Present Imperviousness to PI4KIIIb Inhibitors Additionally Give Imperviousness to ITZ, yet ITZ Does Not Hinder PI4KIIIb Action

As an initial step to recognizing the antiviral focus of ITZ, we considered its impact on replication of CVB3 mutant infections that we beforehand chose for resistance against different inhibitors. CVB3 conveying transformation 3A[H57Y] which presents imperviousness to PI4KIIIb inhibitors (e.g., PIK93, enviroxime, GW5074) (van der Schaar et al., 2012) demonstrated less touchy to ITZ than wildsort (WT) CVB3 in both a solitary cycle replication test and a multicycle CPElessening examine. Different transformations in $3 \mathrm{~A}$ that were appeared to secure against PI4KIIIb inhibitors (i.e., V45A and I54F) (van der Schaar et al., 2012), likewise gave cross-imperviousness to ITZ . Correspondingly, change A70T in PV 3A, which was additionally appeared to secure against PI4KIIIb inhibitors (Arita et al., 2009), ensured PV against ITZ. These outcomes infer a connection between 3A, PI4P lipids, and the instrument of antiviral activity of ITZ.

To figure out if ITZ restrains PI4KIIIb movement, we briefly transfected cells with 


\section{(INTERNATIONAL JOURNAL ONLINE OF SCIENCE)}

\section{ISSN: $2455-0108$}

a hereditarily encoded PI4P sensor i.e. the GFP-labeled PH area of (FAPP1-PH-GFP). Limitation of this sensor particularly relies on upon action of PI4-KIIIb (Hotshot et al., 2005; van der Schaar et al., 2012). In control cells, FAPP1-PH-GFP covered with the Golgi-limited PI4-KIIIb. Upon treatment with a PI4KIIIb inhibitor, PIK93, FAPP1-PH-GFP was redistributed to the cytosol. ITZ, be that as it may, did not diminish FAPP1-PH-GFP confinement. Truth be told, ITZ created a little increment in the measure of Golgi-confined FAPP1PH-GFP, which was more evident in a cell line steadily communicating this PI4P sensor (which demonstrated a more homogenous and moderate expression level). Additionally after recoloring PI4P with a particular neutralizer, a PI4KIIIb inhibitor, BF738735 (van der Schaar et al., 2013), diminished PI4P levels, while ITZ expanded PI4P levels.

CVB3 replication is not totally hindered by ITZ, in this manner allowing the observing of PI4P lipids

\subsection{ITZ Inhibits Virus Replication by}

\section{Targeting OSBP and ORP4}

Having discounted PI4KIIIb as an objective of ITZ, we next swung to flagging strides downstream of PI4P, i.e., proteins that dilemma to PI4P lipids. To evaluate whether any of the known PI4P-restricting proteins could be an objective of ITZ, we performed target recognizable proof by little meddling RNA (siRNA) refinement (TISS) measure (Arita et al., 2010). TISS incorporates siRNA knockdown of applicant target proteins to potentiate the natural impact of a low convergence of a
Volume II, Issue VI SEPTEMBER

compound. Among various PI4Pauthoritative, Golgi-restricted proteins, knockdown of OSBP, however no of the other $\mathrm{PH}$ space containing proteins, improved the inhibitory impact of a low focus (1.25 mM) of ITZ on PV replication, inferring OSBP as a conceivable antiviral focus of ITZ. We facilitate evaluated this probability by a few ex-periments. Initially, the OSBP foe OSW-1 (Burgett et al.,2011) strongly hindered CVB3 replication, affirming that pharmacological focusing of OSBP can repress enterovirus replication. With respect to ITZ, the 3A [H57Y] change in CVB3 gave resistance against OSW-. Much the same as ITZ, OSW-1 hindered all enteroviruses tried and in addition EMCV, however not ERAV (information not appeared). Essentially, OSW-1 did not influence endolysosomal cholesterol conveyance, supporting our past decision that this impact impossible clarifies the antiviral impact of ITZ. Second, comparative concerning PV (Wang et al., 2014), siRNA thump down of OSBP repressed replication of EV71 and HRV2. CVB3 replication was likewise restrained by OSBP thump down, yet this distinction was not measurably noteworthy, in accordance with the lower affectability of CVB3 than EV71 to ITZ. Third, overexpression of OSBP reestablished replication of CVB3 and EV71 within the sight of ITZ or OSW-1, affirming that restraint of viral replication by ITZ and OSW-1 is interceded through OSBP. Overexpression of PI4KIIIb neglected to safeguard replication, and OSBP overexpression did not give salvage against PI4KIIIb inhibitors (information not 


\section{(INTERNATIONAL JOURNAL ONLINE OF SCIENCE)}

\section{ISSN: $2455-0108$}

appeared), demonstrating the specificity of the test setup.

Other than OSBP, OSW-1 additionally targets ORP4 (Burgett et al., 2011). Knockdown of ORP4, however none of alternate ORPs, additionally sharpened PV to ITZ, and overexpression of ORP4 balanced the inhibitory impact of OSW-1 on CVB3 and EV71 replication. We likewise endeavored to test the impact of ORP4 exhaustion. Despite the fact that in the TISS examine, ORP4 thump down potentiated the impact of ITZ, we were not ready to accomplish strong knockdown (>75\% at mRNA level), and accordingly we can't finish up unambiguously whether ORP4 is essential for infection replication. Issues with ORP4 knockdown were additionally seen by others and are likely because of a fundamental part of ORP4 in cell expansion and survival (Charman et al.,2014). All in all, these outcomes show that both OSBP and ORP4 are novel focuses of ITZ and are included in its component of antiviral activity.

\subsection{ITZ Inhibits In Vitro HCV} Replication

Replication of $\mathrm{HCV}$ likewise requires OSBP and is restrained by OSW-1 (Wang et al., 2014). In accordance with our discoveries for enteroviruses, we found that ITZ and posaconazole, yet not the other chose azoles, restrained HCV replication in cell society. EC50 values for restraint of HCV replication by ITZ were similar to those got for the enteroviruses. Together, our information unmistakably shows that ITZ hinders OSBP capacity and that infections from various families that rely on
Volume II, Issue VI SEPTEMBER

upon OSBP capacity can be repressed by ITZ. Vitally, not all (+) RNA infections are touchy to restraint of OSBP. Dengue infection replication was as of late seen to be uncaring to OSW-1 (Wang et al., 2014), and we likewise demonstrated that replication of mouse hepatitis infection (a coronavirus) is unfeeling to OSW-1 and ITZ.

GFP-OSBP fluorescence at the Golgi was plainly expanded in cells treated with either ITZ or OSW-1 and kept on expanding to the detriment of the cytoplasmic sign. OSW-1 was beforehand answered to disturb the struc-ture of the Golgi mechanical assembly (Burgett et al., 2011), which we additionally saw from 30 to 60 min forward as GFP-positive punctae that turned out to be increasingly various after some time. In ITZ-treated cells, the Golgi design got to be influenced just hours after the fact and seemed less scattered than that in OSW-1 treated cells.

\subsection{ITZ Directly Inhibits Lipid Shuttling by OSBP}

To examine whether ITZ can obstruct the lipid exchange action of OSBP, we utilized an arrangement of as a part of vitro liposomal tests (Mesmin et al., 2013) (Supplemental Exploratory Methods) to quantify the vehicle of dehydroergosterol (DHE) and PI4P between ER-like and Golgi-like liposomes. ITZ hindered the sterol-exchange action of cleansed OSBP in a measurement subordinate way with a half inhibitory fixation (IC50) of $200 \mathrm{nM}$. At 1 $\mathrm{mM}$, ITZ and posaconazole, yet not the other chose azoles, firmly restrained DHE move transport in this liposomal examine, 


\section{ISSN: $2455-0108$}

in spite of the fact that they were less powerful than the known OSBP ligand $25 \mathrm{OH}$.

For obscure reasons, a stimulatory impact of $25 \mathrm{OH}$ on PI4P exchange was watched, which relied on upon the $2 \%$ cholesterol substance of the ER-like liposomes. The IC50 values propose that ITZ is more powerful toward sterol than PI4P exchange. Vitally, for specialized reasons, the sterol and PI4P-moving examines are performed under various conditions and in this way can't be specifically analyzed. Further examinations would be expected to build up whether ITZ without a doubt more strongly hinders sterol than PI4P carrying.

ITZ may repress the lipid exchange elements of OSBP specifically by hindering the capacity of the ORD, which exchanges the lipids, or by implication by upsetting the authoritative of OSBP to the liposomes. To explore whether ITZ represses authoritative of OSBP to the liposomes, we examined whether it meddles with the connections between (1) the FFAT-theme and VAP-An on the ER-like liposomes and (2) the PHspace and PI4P on the Golgi-like liposomes. To this end, we performed liposomal skim up examinations utilizing a recombinant part of OSBP containing the $\mathrm{PH}$ area and FFAT theme (amino acids 76-408; PHFFAT). Within the sight of VAP-A, PHFFAT bound to the ER-like lipo-somes, and this collaboration was not upset by $1 \mathrm{mM}$ ITZ. The communication of PH-FFAT with PI4P-containing Golgi-like liposomes was not disturbed by $10 \mathrm{mM}$ ITZ either. In like manner, VAP-A communication with $\mathrm{PH}-$
Volume II, Issue VI SEPTEMBER

FFAT selected to Golgi-like liposomes was additionally coldhearted to $10 \mathrm{mM}$ ITZ. Together, the liposomal coast up measures demonstrate that ITZ does not meddle with the official of OSBP to the liposomes by means of VAP-An and PI4P.

To set up whether ITZ hinders the lipid exchange action of the ORD, we made utilization of a formerly settled test (Mesmin et al., 2013). Constrained tryptic proteolysis of OSBP severs OSBP into three noteworthy pieces; a $43 \mathrm{kDa}$ section containing the PH-space and FFAT-theme, and two parts of $35 \mathrm{kDa}$ and $20 \mathrm{kDa}$ that are gotten from the ORD. Already, it was demonstrated that the ORD-determined sections hold lipid exchange action, additionally without the idle $43 \mathrm{kDa}$ piece (Mesmin et al., 2013). We observed that ITZ still restrained both DHE (Figure 5K) and PI4P exchange by OSBP that had been subjected to tryptic proteolysis. These outcomes recommend that ITZ represses both the sterol and PI4P-exchange exercises of OSBP by focusing on the ORD.

\subsection{ITZ Binds Directly to OSBP}

The inhibitory impact of ITZ on OSBP capacity in an insignificant in vitro framework suggested that ITZ specifically hinders OSBP. To biochemically characterize the authoritative in more detail; we gauged official of ITZ to GFP-OSBP utilizing microscale thermophoresis (MST). Every atom or complex conveys diversely in a temperature field, contingent upon size, charge, and the hydration shell. Authoritative of ITZ to OSBP will influence the hydration shell and in this way its thermophoretic conduct. ITZ modified 


\section{(INTERNATIONAL JOURNAL ONLINE OF SCIENCE)}

\section{ISSN: $2455-0108$}

the thermophoretic profiles of refined GFPOSBP in a measurement depen-scratch way, showing direct authoritative. Standardization and fitting of information from three autonomous estimations evil presence started that ITZ ties to OSBP with a KD of $430 \mathrm{nM}$. The monophasic state of the coupling bend shows that there is likely just a solitary restricting site for ITZ on OSBP, in spite of the fact that our information can't decide out that there are two locales with almost indistinguishable KD's.

\subsection{ITZ Inhibits PI4P and Cholesterol}

\section{Shuttling at ROs}

To test specifically whether ITZ represses the PI4P carrying capacity of OSBP at ROs, cells were contaminated with CVB3 and repli-cation was permitted to advance uninhib-ited for $3 \mathrm{hr}$. At that point ITZ or BF738735 were included for $1 \mathrm{hr}$, cells were handled for microscopy, and PI4P power at ROs was evaluated. ITZ treatment brought about a solid increment in PI4P signal at the ROs (half increment), though BF738735 treatment decreased it by half, in accordance with the impacts of these meds on OSBP enrollment. No such impacts on PI4P were seen upon treatment with guanidine, an inhibitor of the viral $2 \mathrm{C}$ protein, which was incorporated to decide out that the watched impacts were just because of a hindrance of replication. In this way, these outcomes show that in tainted cells, ITZ keeps the expulsion of PI4P from ROs, which is tantamount to our perceptions in uninfected cells.

To test whether ITZ additionally represses cholesterol transporting to ROs,
Volume II, Issue VI SEPTEMBER

cells were tainted and regarded comparable as portrayed above, cholesterol was pictured by filipin recoloring, and colocalization of filipin with $3 \mathrm{~A}$ was evaluated utilizing a Pearson's connection co-productive. In DMSO-treated cells, filipin halfway covered with 3A (Pearson's 0.53). ITZ essentially diminished the colocalization of filipin with 3A (Pearson's 0.38), showing that ITZ repressed the redistribution of cholesterol to the ROs. Likewise, BF738735, which decreases the restriction of OSBP to ROs, additionally hindered cholesterol transporting to ROs.

\section{DISCUSSION}

Enteroviruses change cell lipid homeostasis and rebuild host cell films into replication organelles by usurping various host proteins, for example, PI4KIIIb (Arita et al., 2011; Hsu et al., 2010). In any case, up 'til now little is thought about the hidden instruments and the personality of other host components included. Illustration of the system of activity of inhibitors of infection replication has demonstrated instrumental in acquiring novel bits of knowledge into the components of viral replication. In this study we recognized ITZ, a generally utilized antifungal medication that is as of now likewise being investigated as an anticancer specialist, as a novel, expansive range inhibitor of enteroviruses, cardioviruses, and HCV. We demonstrate that none of the entrenched focuses of ITZ (i.e., hCYP51, mTOR, VEGFR2, Hh) clarifies its antiviral action. Rather, we recognized the PI4P-restricting proteins OSBP and ORP4 as novel focuses 


\section{(INTERNATIONAL JOURNAL ONLINE OF SCIENCE)}

\section{ISSN: $2455-0108$}

of ITZ through which the antiviral impact is intervened.

OSBP is an expert controller of lipid homeostasis at MCSs be-tween the ER and the trans-Golgi device. It trades cholesterol and PI4P between these films and has been proposed to control MCS dependability (Mesmin et al., 2013). OSBP is the model individual from the group of ORPs, a gathering of proteins whose phone capacities have remained inadequately caught on. We recognized OSBP and ORP4 as focuses of ITZ. Pharmacologic restraint, siRNA knockdown, and salvage of replication by over-expression exhibit the significance of these proteins for infection replication. Besides, OSBP confined to ROs in a PI4-KIIIb-and PI4P-subordinate way. ITZ straightforwardly bound cleaned OSBP and hindered both the cholesterol and PI4Ptransport exercises of OSBP in vitro (in liposomal tests). Additionally in living (uninfected) cells, ITZ repressed the vehicle capacity of OSBP (i.e., transport of cholesterol from ER to Golgi and transport of PI4P from Golgi to ER), prompting an expansion in PI4P levels at the Golgi, in this way bringing about the aggregation of OSBP. In like manner, in tainted cells, ITZ expanded PI4P levels on ROs, again prompting an upgraded enlistment of OSBP, and hindered the amassing of cholesterol on ROs. Consequently, we show that ITZ represses the lipid-carrying elements of OSBP in vitro as well as in both tainted and uninfected cells.

The enteroviral proteins $2 \mathrm{BC}$ and $3 \mathrm{~A}$ assume a basic part in RO arrangement by
Volume II, Issue VI SEPTEMBER

enlisting PI4KIIIb, which prompts the gathering of PI4P lipids on ROs (Arita, 2014; Arita et al., 2011; Hsu et al., 2010). We here demonstrate that OSBP is hence enrolled to ROs by means of PI4P. Our information show that at ER-RO MCSs, OSBP trades PI4P for cholesterol, either recently blended in the ER or starting from a lipid bead stockpiling pool and being activated through the ER, prompting a gathering of cholesterol at the ROs (Arita, 2014). Our discoveries are in concurrence with those of a late paper that proposed that OSBP transports cholesterol to HRV ROs taking into account the inhibitory impacts on HRV replication of OSBP knockdown and $25 \mathrm{OH}$ treatment (Roulin et al., 2014). The finding that the levels of cholesterol are hoisted to the detriment of cholesterylesters (i.e., the structure in which cholesterol is put away in lipid beads) in enteroviruscontaminated cells (Ilnytska et al., 2013; Roulin et al., 2014) recommends that put away cholesterol is activated for transport to ROs. Also, uptake of cholesterol by endo-cytosis has been recommended to add to the amassing of cholesterol at ROs (Ilnytska et al., 2013). The part of cholesterol collection at ROs is a long way from built up. Cholesterol is of significant significance for films properties, for example, layer ease and development of lipid microdomains, and itis along these lines likely imperative for the layer modifications and distortions fundamental RO arrangement. Furthermore, cholesterol modifications have been recommended to influence viral polyprotein preparing productivity (Ilnytska et al., 2013). 


\section{(INTERNATIONAL JOURNAL ONLINE OF SCIENCE)}

\section{ISSN: $2455-0108$}

The movement of OSBP is likewise vital for the homeostasis of different lipids. At ER-Golgi MCSs, it acts working together with the PI exchange protein Nir2, which supplies PI for PI4P union at Golgi layers, and CERT, which exchanges ceramide to Golgi for sphingomyelin combination, in this manner creating diacylglycerols (DAGs) (Peretti et al., 2008). Significantly OSBP ligands, e.g.,25OH and OSW-1, change the confinement of CERT and adjust sphingomyelin amalgamation (Burgett et al., 2011; Perry and Ridg-way, 2006). As an inhibitor of OSBP-intervened lipid carrying, ITZ may consequently influence the amassing of cholesterol as well as bother the homeostasis of different lipids, for example, sphingomyelin and DAGs. Whether and how this adds to the restraint of RO development and/or capacity stays to be set up.

\section{EXPERIMENTAL Methods}

Insights about distributed and standard strategies (cell society, plasmids, infection in-fections, replicon transfections, the TISS measure, salvage tests, investigation of viral polyprotein handling, siRNA tests, immunofluorescence mi-croscopy, and liposomal examines) are given in Supplemental Test Methodology.

\subsection{Reagents}

The accompanying mixes were acquired: itraconazole (Santa Clause Cruz Biotechnology); posaconazole (Merck); ketoconazole (Enzo Life Sciences); flucona-zole and voriconazole (Pfizer); T00127-HEV1 (Pharmeks); dipyridamole,
Volume II, Issue VI SEPTEMBER

gua-nidine hydrochloride ( $\mathrm{GuHCl})$, and bestradiol (Sigma Aldrich); Sant-1, Sant-2 (Tocris Bioscience); and cyclopamineKAAD (Calbiochem). PIK93 was a kind blessing from Dr. K. Shokat (Universeity of California, Berkeley), BF738735 (Mama cLeod et al., 2013) was given by Galapagos NV, and OSW-1 was detached from nature (Burgett et al., 2011). B-Estradiol was disintegrated by producer's guidelines. $\mathrm{GuHCl}$ was broken up in water and all other com-pounds in DMSO.

\subsection{Compound Library Screen}

The NIH Clinical Gathering was acquired from the NIH. The 446 exceptionally sedate like mixes were screened for inhibitors of CVB3 utilizing lessening of CPE as readout. Subconfluent monolayers of Wild ox green monkey kidney (BGM) cells in 96-well plates were contaminated with 10 CCID50 of CVB3 per well, mixes were added to a last grouping of $10 \mathrm{mM}$, and the level of CPE was outwardly evaluated following 2 days of brooding at $37 \mathrm{C}$ when full CPE had created in the tainted, untreated control wells.

\subsection{Live-Cell Imaging}

For live-cell imaging tests, HeLa R19 cells were transfected with pEGFP-hOSBP; treated with ITZ, OSW-1, or dissolvable control (DMSO); and imaged utilizing a Nikon A1R confocal laser checking magnifying lens. Pictures were handled and measured utilizing the Nikon NISComponents programming. For extra subtle 
(INTERNATIONAL JOURNAL ONLINE OF SCIENCE)

ISSN: $2455-0108$

elements, see supplemental Trial Methodology.

\subsection{Microscale Thermophoresis}

The collaboration amongst ITZ and recombinant GFP-hOSBP-SII (human OSBP with a N-terminal GFP and a Cterminal Strep-tagII) was examined by MST utilizing a NanoTemper Stone monument NT.115 instrument and the NTAnalysis programming

(NanoTemper

Advancements).

\section{REFERENCES}

1. Arita, M. (2014). Phosphatidylinositol-4 kinase III beta and oxysterol-binding protein accumulate unesterified cholesterol on poliovirus-induced membrane structure. Microbiol. Immunol. 58, 239-256.

2. Arita, M., Takebe, Y., Wakita, T., and Shimizu, H. (2010). A bifunctional antienterovirus compound that inhibits replication and the early stage of enterovirus 71 infection. J. Gen. Virol. 91, 2734-2744.

3. Arita, M., Kojima, H., Nagano, T., Okabe, T., Wakita, T., and Shimizu, H. (2011). Phosphatidylinositol 4-kinase III beta is a target of enviroxime-like compounds for antipoliovirus activity. $\mathbf{J}$. Virol. 85, 2364-2372.

4. Balla, A., Tuymetova, G., Tsiomenko, A., Va' rnai, P., and Balla, T. (2005). A plasma membrane pool of phosphatidylinositol 4-phosphate is
Volume II, Issue VI SEPTEMBER

generated by phosphatidylinositol 4kinase type-III alpha: studies with the $\mathrm{PH}$ domains of the oxysterol binding protein and FAPP1. Mol. Biol. Cell 16, 1282-1295.

5. Belov, G.A., Nair, V., Hansen, B.T., Hoyt, F.H., Fischer, E.R., and Ehrenfeld, E. (2012). Complex dynamic development of poliovirus membranous replication complexes. J. Virol. 86, 302312.

6. Beretta, L., Svitkin, Y.V., and Sonenberg, N. (1996). Rapamycin stimulates viral protein synthesis and augments the shutoff of host protein synthesis upon picornavirus infection. J. Virol. 70, 8993-8996.

7. Burgett, A.W., Poulsen, T.B., Wangkanont, K., Anderson, D.R., Kikuchi, C., Shimada, K., Okubo, S., Fortner, K.C., Mimaki, Y., Kuroda, M., et al. (2011). Natural products reveal cancer cell dependence on oxysterolbinding pro- teins. Nat. Chem. Biol. 7, 639-647.

8. Charman, M., Colbourne, T.R., Pietrangelo, A., Kreplak, L., and Ridgway, N.D. (2014). Oxysterolbinding protein (OSBP)-related protein 4 (ORP4) is essential for cell proliferation and survival. J. Biol. Chem. 289, 15705-15717.

9. Chen, J.K., Taipale, J., Young, K.E., Maiti, T., and Beachy, P.A. (2002). Small molecule modulation of 


\section{(INTERNATIONAL JOURNAL ONLINE OF SCIENCE)}

\section{ISSN: $2455-0108$}

Smoothened activity. Proc. Natl. Acad. Sci. USA 99, 14071-14076.

10. Doedens, J.R., Giddings, T.H., Jr., and Kirkegaard, K. (1997). Inhibition of endoplasmic reticulum-to-Golgi traffic by poliovirus protein $3 \mathrm{~A}$ : genetic and ul- trastructural analysis. J. Virol. 71, 9054-9064.

11. Hsu, N.-Y., Ilnytska, O., Belov, G., Santiana, M., Chen, Y.-H., Takvorian, P.M., Pau, C., van der Schaar, H., Kaushik-Basu, N., Balla, T., et al. (2010). Viral reor- ganization of the secretory pathway generates distinct organelles for RNA replication. Cell 141, 799-811.

12. Ilnytska, O., Santiana, M., Hsu, N.Y., Du, W.L., Chen, Y.H., Viktorova, E.G., Be- lov, G., Brinker, A., Storch, J., Moore, C., et al. (2013). Enteroviruses harness the cellular endocytic machinery to remodel the host cell cholesterol land- scape for effective viral replication. Cell Host Microbe 14, 281-293.

13. Kim, J., Tang, J.Y., Gon, R., Kim, J., Lee, J.J., Clemons, K.V., Chong C.R., Chang, K.S., Fereshteh, M., Gardner, D., et al. (2010). Itraconazole, a commonly used antifungal that inhibits Hedgehog pathway activity and cancer growth. Cancer Cell 17, 388-399.

\section{Volume II, Issue VI SEPTEMBER}

14. Kim, D.J., Kim, J., Spaunhurst, K., Montoya, J., Khodosh, R., Chandra, K., Fu, T., Gilliam, A., Molgo, M., Beachy, P.A., and Tang, J.Y. (2014). Open-label, exploratory phase II trial of oral itraconazole for the treatment of basal cell car- cinoma. J. Clin. Oncol. 32, 745-751.

15. Lestner, J., and Hope, W.W. (2013). Itraconazole: an update on pharmacology and clinical use for treatment of invasive and allergic fungal infections. Expert Opin. Drug Metab. Toxicol. 9, 911-926.

16. Limpens, R.W.A.L., Van der Schaar, H.M., Kumar, D., Koster, A.J., Snijder, E.J., Van Kuppeveld, F.J.M., and $\mathrm{Ba}^{\prime}$ rcena, M. (2011). The transformation of enterovirus replication structures: a threedimensional study of single- and double-membrane compartments. MBio. 2, e00166-11.

17. MacLeod, A.M., Mitchell, D.R., Palmer, N.J., Van de Poe" 1, H., Conrath, K., An- drews, M., Leyssen, P., and Neyts, J. (2013). Identification of a series of compounds with potent antiviral activity for the treatment of enterovirus infections. ACS Med. Chem. Lett. 4, 585-589.

18. Mesmin, B., Bigay, J., Moser von Filseck, J., Lacas-Gervais, S., Drin, G., and Antonny, B. (2013). A fourstep cycle driven by PI(4)P hydrolysis 
(INTERNATIONAL JOURNAL ONLINE OF SCIENCE)

\section{ISSN: $2455-0108$}

directs sterol/ PI(4)P exchange by the

ER-Golgi tether OSBP. Cell 155, 830-843.

19. Nacev, B.A., Grassi, P., Dell, A., Haslam, S.M., and Liu, J.O. (2011). The anti- fungal drug itraconazole inhibits vascular endothelial growth factor receptor 2 (VEGFR2) glycosylation, trafficking, and signaling in endothelial cells. J. Biol. Chem. 286, 44045-44056.

20. Peretti, D., Dahan, N., Shimoni, E., Hirschberg, K., and Lev, S. (2008). Coordi- nated lipid transfer between the endoplasmic reticulum and the Golgi complex requires the VAP proteins and is essential for Golgimediated transport. Mol. Biol. Cell 19, 3871-3884.

21. Perry, R.J., and Ridgway, N.D. (2006). Oxysterol-binding protein and vesicle- associated membrane proteinassociated protein are required for sterol- dependent activation of the ceramide transport protein. Mol. Biol. Cell 17,2604-2616.

22. Raychaudhuri, S., and Prinz, W.A. (2010). The diverse functions of oxysterol- binding proteins. Annu. Rev. Cell Dev. Biol. 26, 157-177.

23. Rothwell, C., Lebreton, A., Young Ng, C., Lim, J.Y., Liu, W., Vasudevan, S., Labow, M., Gu, F., and Gaither, L.A. (2009). Cholesterol biosynthesis modula- tion regulates
Volume II, Issue VI SEPTEMBER

dengue viral replication. Virology $389,8-19$.

24. Roulin, P.S., Lo“ tzerich, M., Torta, F., Tanner, L.B., van Kuppeveld, F.J., Wenk, M.R., and Greber, U.F. (2014). Rhinovirus uses a phosphatidylinositol 4-phosphate/cholesterol counter-current for the formation of replication compartments at the ER-Golgi interface. Cell Host Microbe 16, 677-690.

25. Rudin, C.M., Brahmer, J.R., Juergens, R.A., Hann, C.L., Ettinger, D.S., Sebree, R., Smith, R., Aftab, B.T., Huang, P., and Liu, J.O. (2013). Phase 2 study of pe- metrexed and itraconazole as second-line therapy for metastatic nonsqua- mous nonsmall-cell lung cancer. J. Thorac. Oncol. 8, 619-623.

26. Taipale, J., Chen, J.K., Cooper, M.K., Wang, B., Mann, R.K., Milenkovic, L., Scott, M.P., and Beachy, P.A. (2000). Effects of oncogenic mutations in Smoothened and Patched can be reversed by cyclopamine. Nature 406, 1005-1009.

27. Van der Schaar, H.M., van der Linden, L., Lanke, K.H., Strating, J.R., Pu“ r- stinger, G., de Vries, E., de Haan, C.A., Neyts, J., and van Kuppeveld, F.J. (2012). Coxsackievirus mutants that can bypass host factor PI4KIIIb and the need for high levels of PI4P lipids for replication. Cell Res. 22, 1576-1592. 
28. Van der Schaar, H.M., Leyssen, P., Thibaut, H.J., de Palma, A., van der Linden, L., Lanke, K.H., Lacroix, C., Verbeken, E., Conrath, K., Macleod, A.M., et al. (2013). A novel, broadspectrum inhibitor of enterovirus replication that targets host cell factor phosphatidylinositol 4-kinase IIIb. Antimicrob. Agents Chemo- ther. 57, 4971-4981.

29. Van Kuppeveld, F.J., Galama, J.M., Zoll, J., and Melchers, W.J. (1995). Genetic analysis of a hydrophobic domain of coxsackie B3 virus protein 2B: a moder- ate degree of

31. Warrilow, A.G., Parker, J.E., Kelly, D.E., and Kelly, S.L. (2013). Azole affinity of sterol 14a-demethylase (CYP51) enzymes from Candida albicans and Homo sapiens. Antimicrob. Agents Chemother. 57, 1352-1360.

32. Weber-Boyvat, M., Zhong, W., Yan, D., and Olkkonen, V.M. (2013). Oxysterol- binding proteins: functions in cell regulation beyond lipid metabolism. Bio- chem. Pharmacol. 86, 89-95.

33. Wyles, J.P., Perry, R.J., and Ridgway, N.D. (2007). Characterization of the sterol-binding domain of oxysterolbinding protein (OSBP)-related protein 4 reveals a novel role in vimentin organization. Exp. Cell Res. 313, 1426-1437. hydrophobicity is required for a cisacting function in viral RNA synthesis. J. Virol. 69, 7782-7790.

30. Walsky, R.L., Bauman, J.N., Bourcier, K., Giddens, G., Lapham, K., Negahban, A., Ryder, T.F., Obach, R.S., Hyland, R., and Goosen, T.C. (2012). Optimized assays for human UDP-glucuronosyltransferase (UGT) activities: altered ala- methicin concentration and utility to screen for UGT inhibitors. Drug Metab. Dispos. 40, 1051-1065. 